\title{
Ethical Considerations of Photosensitive Epilepsy in Mixed Reality
}

Laura South

Northeastern University

Boston, MA 02115, USA

south.|@husky.neu.edu
Michelle A. Borkin

Northeastern University

Boston, MA 02115, USA

m.borkin@northeastern.edu

\begin{abstract}
As public interest in virtual and augmented reality increases, so do the risks faced by users with photosensitive epilepsy, a neurological condition where seizures are triggered by specific kinds of light stimuli. Most research on photosensitive epilepsy focuses on user interaction with television sets and does not necessarily transfer to other methods of interaction. Very little research has been done examining mixed reality from the perspective of photosensitive users and understanding the additional risks posed by new forms of immersive technology. Examples of hackers targeting people with photosensitive epilepsy through social media serve as a wake-up call about the possibility of similar malicious attacks in mixed reality. In this paper we draw from photosensitive epilepsy research to create recommendations for simple steps mixed reality developers can take to minimize photosensitive risk in their systems in addition to issuing a broader call for further research into understanding photosensitive epilepsy in the context of mixed reality.
\end{abstract}

\section{Author Keywords}

accessibility; mixed reality; epilepsy

\section{CCS Concepts}

-Human-centered computing $\rightarrow$ Mixed / augmented reality; Accessibility theory, concepts and paradigms; 


\section{Introduction}

Epilepsy is the most common serious neurological condition in the world, affecting nearly 70 million people worldwide [5]. Between 2 and $14 \%$ of people with epilepsy will experience seizures precipitated by specific sequences of light stimuli [6]. This form of epilepsy is known as "photosensitivity" or "photosensitive epilepsy".

There are three kinds of light stimuli sequences that are likely to induce seizures when viewed by someone with photosensitive epilepsy: flashes, transitions to and from saturated red, and repeated striped patterns [6]. Flashing sequences are likely to be dangerous if they contain more than 3 flashes per second [4]. Similarly, red-light flicker at wavelengths of $660-720 \mathrm{~nm}$ is considered dangerous if more than 3 transitions are observed per second [3]. These sequences are more likely to trigger seizures than blue or white light of the same overall intensity. Finally, stationary repeated stripe patterns can induce seizures if more than eight clearly distinguishable light-dark pairs are visible [15].

In everyday life exposure to flashing lights and other seizureinducing sequences can be minimized by avoiding places where these sequences are likely to occur, such as concerts [12], movie theaters [11], and even areas with wind turbines [8]. Innovations like social media and mixed reality alter the equation by a) increasing the likelihood of accidental exposure to seizure-inducing sequences and b) enabling attackers to directly target people with photosensitive epilepsy for a variety of motivations, including cyberbullying, intimidation, or a more general desire to inflict pain of some kind. In this paper, we summarize trends in the relationship between photosensitive epilepsy and technology, recommend accessibility best practices for developers and researchers in mixed reality, and issue a broader call for further research into the intersection of immersive techonology

\section{and photosensitivity.}

\section{Photosensitive epilepsy: then and now}

In 1997, an episode of the television show Pokemon containing a sequence of red and blue flashing lights was aired in Japan, causing hundreds of children to suffer seizures or other forms of photosensitive stress [13]. In 2016, 19 years later, a journalist who had written publicly about his lived experiences with photosensitive epilepsy and extensively critiqued then-U.S. Presidential candidate Donald Trump was sent a strobe GIF on Twitter with the message "You deserve a seizure for your posts" [9]. These two instances, although similar in their unfortunate repercussions, represent a shift that has occurred in the last twenty years in the relationship between photosensitive epilepsy and technology.

In the 1990s and early 2000s, photosensitive harm from technology followed the same template as the Pokemon incident: a sequence with epileptogenic potential is inadvertently included in an otherwise harmless piece of media which is broadcast widely and eventually is seen by someone with photosensitive epilepsy, who suffers from some form of photosensitive stress in response. After the Pokemon incident, a flurry of research papers were published describing medical trials done to isolate the specific factors that contribute to seizure-inducing potential. Soon software solutions like the Harding Flash and Pattern Analyzer ${ }^{1}$ and the Photosensitive Epilepsy Analysis Tool ${ }^{2}$ were built to apply the findings of these papers and allow content creators to programmatically identify sequences with epileptogenic content. This neatly solved the problem of accidentally airing seizure-inducing content on television, but did not address the possibility of intentionally seizure-inducing content being sent directly to people with photosensitive epilepsy.

\footnotetext{
${ }^{1}$ https://www.hardingfpa.com/

${ }^{2}$ https://trace.umd.edu/peat
} 
A new form of photosensitive risk began to evolve in the 2010s. People with photosensitive epilepsy are no longer stumbling upon seizure-inducing sequences by unfortunate chance; they are being explicitly targeted by flashing content created for the specific purpose of causing photosensitive harm. The attacks take different forms beyond the direct one-to-one targeting exhibited in the journalist attack: in 2008 hackers inserted strobe GIFs and videos into the Epilepsy Foundation website with the assumed intention of causing indiscriminate harm to all viewers with photosensitive epilepsy [10]. The Epilepsy Foundation Twitter account was hacked and began posting strobing GIFs in December 2019, causing an unknown amount of harm to any Twitters users with photosensitive epilepsy who happened to be following the EF's account and checking their timeline [1]. Multiple posts on the r/epilepsy subreddit ${ }^{3}$ ask for help with avoiding intentionally seizure-inducing content online and reporting senders to the police.

\section{Photosensitive epilepsy in mixed reality}

Mixed reality has not yet reached the level of intentional and targeted photosensitive attacks, but instances of accidentally triggered seizures in mixed reality have already begun to occur. In January 2018, a player in the popular virtual reality online world VRChat suffered a photosensitive seizure while wearing a full-body tracking suit, allowing nearby players to witness his convulsions firsthand [2]. After several minutes, bystanders were eventually able to draw a moderator's attention to the situation and a welfare check was called in to the local police. As a result, players began lobbying game creators to include a "medical alert" button for future emergencies. Four months later, in April, another player had a photosensitive seizure in VRChat. This time, the entire episode was recorded and later posted on YouTube by another player, who claims that bystanders

\footnotetext{
${ }^{3}$ https://www.reddit.com/r/Epilepsy/
}

attempted to contact moderators about the situation for over a hour [14]. The seizing player was eventually identified by the surrounding players, and Toronto police were alerted about the situation and were able to check in on the player, who fully recovered.

The VRChat community has generally been supportive in these cases of medical emergencies, going above and beyond to ensure that the player suffering from the seizure receives the help they need. The attitudes of VR enthusiasts not currently involved in active medical emergencies are not always so supportive. Immediately following the January 2018 seizure, a VRChat player proposed a feature request asking for seizure-detection that would alert moderators if a player's headset fell to the ground. Another player responded to this request by saying "If VRchat adds in an option to enable a warning when you're on the floor for example then all this is going to do is encourage people who do have problems to play the game using that as a bit of a failsafe, it could actually cause more harm than good." The implication of this comment is that people with photosensitive epilepsy should avoid VR entirely, and that protective measures shouldn't be added for fear that they would encourage people with photosensitivity to participate in VR. Unfortunately photosensitive epilepsy is a condition that is often not diagnosed until a patient has experienced their first seizure [6]. It is not enough to discourage anyone who might have photosensitive epilepsy from participating in mixed reality - the field needs to be proactive in building protections against seizure-inducing content.

Most seizures reported in mixed reality are the result of accidental exposure rather than explicit targeting, but it is easy to imagine how the recent trend observed in social media of

${ }^{4}$ https://vrchat.canny.io/feature-requests/p/epilepsy-detection 
targeting people with photosensitive epilepsy could be replicated, possibly to a more dangerous degree, in mixed reality. There are several reasons why content viewed through virtual or mixed reality headsets might be more likely to induce seizures than the same content viewed on laptops or mobile phones. Medical trials have shown that the area of visual cortex to which the content is projected has a positive correlation with likelihood of causing a seizure [4]. Virtual reality headsets are worn close to the eyes and therefore a flashing sequence would affect a greater area of the visual cortex than the same sequence viewed on a television or mobile device.

\section{Next steps and recommendations}

The television industry provides a blueprint that mixed reality researchers can learn from when it comes to improving accessibility for people with photosensitive epilepsy. In the five years after a Pokemon episode containing an accidentally seizure-inducing sequence was broadcast on television, a host of studies were completed identifying photosensitive risk factors $[4,6,15]$. These studies were in turn used to develop software to detect seizure-inducing content in television programs. This incident also led to the development of broadcast guidelines to govern flashes and repeated patterns [7]. Most of the studies used to establish dangerous thresholds measured EEG response while participants interacted a television located several feet away. It is unclear if these thresholds are appropriate for other interaction methods, like mobile phones, laptops, and mixed reality. No studies have been done to assess the impact of mixed reality on photosensitive stress, leaving people with photosensitive epilepsy in limbo when it comes to participating in mixed reality technology. Researchers need to adopt a similar proactive approach when it comes to examining the risks of photosensitive epilepsy in mixed reality environments.
New guidelines and thresholds for mixed reality will take time and effort to create. In the meantime, small improvements on the side of mixed reality creators could significantly improve accessibility for people with photosensitive epilepsy, such as allowing users to apply various filters to their headset views to counteract different harmful sequences. A blue filter has been shown to lower photosensitive response to saturated red transitions, and is a relatively simple feature for developers to add. Similarly, allowing users to easily change contrast and brightness settings could help users quickly neutralize a flashing sequence or patterns with high contrast. Finally, social platforms that incorporate mixed reality, like VRChat, could implement emergency moderator call buttons so nearby players can take action to help players that are having seizures or any number of other possible medical emergencies during gameplay.

\section{Conclusion}

As we consider the ethical dimensions of mixed and virtual reality, it is imperative that we consider accessibility for people with photosensitive epilepsy. It is not sufficient to ask people with photosensitivity to remove themselves from any technology involving mixed reality. As mixed reality becomes more accepted by the general public, it will become harder for people with photosensitive epilepsy to "opt out" of an entire field of technology. By following our recommendations for small steps towards accessibility and by encouraging deeper research into mixed reality and photosensitivity, both developers and researchers can help move the field of mixed reality towards safety and accessibility for all users.

\section{Acknowledgements}

This material is based upon work supported by the National Science Foundation Graduate Research Fellowship. 


\section{REFERENCES}

[1] Jackie Aker. 2019. Epilepsy Foundation Files Criminal Complaint and Requests Investigation in Response to Attacks on Twitter Feed. (2019).

https://www.epilepsy.com/release/2019/12/ epilepsy-foundation-files-criminal-complaintand-requests-investigation-response

[2] Julia Alexander. 2018. After VRChat player suffers seizure, exec says there are ways to help. (2018). https://ww.polygon.com/2018/1/19/16911524/ vrchat-seizure-mod-youtube-rogue-shadow

[3] Joydeep Bhattacharya. 2010. Seeing Red: Colour Modulation and Photosensitive Epilepsy. Epilepsy Professional 18 (2010), 26-29.

[4] CD Binnie, J Emmett, P Gardiner, GFA Harding, D Harrison, and AJ Wilkins. 2002. Characterizing the Flashing Television Images that Precipitate Seizures. SMPTE journal 111, 6-7 (2002), 323-329.

[5] Martin J Brodie, Steven C Schachter, and Patrick Kwan. 2012. Fast Facts: Epilepsy. Karger Medical and Scientific Publishers.

[6] Robert S Fisher, Graham Harding, Giuseppe Erba Gregory L Barkley, and Arnold Wilkins. 2005. Photicand Pattern-induced Seizures: a Review for the Epilepsy Foundation of America Working Group. Epilepsia 46, 9 (2005), 1426-1441.

[7] GFA Harding and PF Harding. 1999. Televised Material and Photosensitive Epilepsy. Epilepsia 40 (1999), 65-69.

[8] Graham Harding, Pamela Harding, and Arnold Wilkins. 2008. Wind Turbines, Flicker, and Photosensitive
Epilepsy: Characterizing the Flashing That May Precipitate Seizures and Optimizing Guidelines to Prevent Them. Epilepsia 49, 6 (2008), 1095-1098.

[9] C Kang. 2017. A Tweet to Kurt Eichenwald, a Strobe, and a Seizure. Now, an Arrest. The New York Times 17 (2017).

[10] Kevin Poulsen. 2008. Hackers Assault Epilepsy Patients via Computer. (2008).

https://www.wired.com/2008/03/hackers-assaultepilepsy-patients-via-computer/

[11] Manish Prasad, Michelle Arora, Ishaq Abu-Arafeh, and Graham Harding. 2012. 3D Movies and Risk of Seizures in Patients with Photosensitive Epilepsy. Seizure 21, 1 (2012), 49-50.

[12] Newel Salet, Marieke Visser, Cornelis Stam, and Yvo M Smulders. 2019. Stroboscopic Light Effects During Electronic Dance Music Festivals and Photosensitive Epilepsy: A Cohort Study and Case Report. BMJ open 9, 6 (2019).

[13] Takeo Takahashi and Yasuo Tsukahara. 1998. Pocket Monster Incident and Low Luminance Visual Stimuli: Special Reference to Deep Red Flicker Stimulation. Pediatrics International 40, 6 (1998), 631-637.

[14] Wikstream. 2018. Seizure in VRChat - REAL Medica Emergency in VR. (2018) https : / / www.youtube.com/watch?v=Ulj4kwGgiQo

[15] Arnold Wilkins, John Emmett, and Graham Harding. 2005. Characterizing the Patterned Images that Precipitate Seizures and Optimizing Guidelines to Prevent Them. Epilepsia 46, 8 (2005), 1212-1218. 